\title{
Estrategias fiscales aplicadas a los modelos de compensación laboral de las empresas transnacionales (Fiscal strategies applied to the labor compensation models of transnational companies)
}

\section{Oscar Tolentino}

\begin{abstract}
Today, transnational companies are faced not only with domestic, but also with international competitors. To offer cost-effective professional services, organizations are required to act on subjects such as pricing models, cost structures, production processes, technological platforms, among others. In the case of Mexico, we can find cost-cutting such as outsourcing, compensation through professional fees, and others. However, what is perhaps one of the most complex structural strategies is to redesign the wages and benefits of employees, to get a model that has a tax benefit for the organization, lightening payroll tax. Under this reality, the design and implementation of such fiscal strategies constitute a factor to be considered for the local economic future and the welfare of the economically active population.
\end{abstract}

Key words: compensation, fiscal strategies, retirement

JEL: J33, M52

Tecnologico de Monterrey, Escuela de Ciencias Sociales y Gobierno, Ave. Eugenio Garza Sada 2501, Monterrey, N.L., México, 64849. oscar.tolentino@gmail.com

El autor agradece el valioso apoyo de la Dra. Bonnie J. Palifka en esta investigación. 
Resumen. En la actualidad, las empresas transnacionales se enfrentan con competidores nacionales e internacionales. Para poder ofertar servicios profesionales eficientes en costos, las organizaciones se ven ante la necesidad de tomar medidas con respecto a temas como sus modelos de precios, estructuras de costos, procesos productivos, plataformas tecnológicas, entre otros. En el caso de México, observamos estrategias como outsourcing, la compensación a través de honorarios profesionales, entre otras. Sin embargo, una de las opciones que pareciera más compleja en su estructura, es la de rediseñar el paquete de compensación, beneficios y prestaciones de los empleados, para conseguir un modelo que tenga un beneficio fiscal para la empresa. Bajo esta realidad, el diseño e implementación de tales estrategias fiscales constituyen un factor a considerar para el futuro económico local y el bienestar patrimonial de la población económicamente activa.

Palabras clave: compensación laboral, estrategias fiscales, retiro

\section{Introducción}

En el mundo globalizado de hoy en día, las empresas con operaciones transnacionales se enfrentan en una dura competencia, ya que deben estar listas para competir por todo tipo de recursos financieros, contra todo el mundo, desde todas partes (Sirkin, Hemerling, Bhattacharya, \& Butman, 2008). No es de extrañar que una de las principales metas que buscan las empresas en el escenario globalizado, es conseguir los atractivos contratos multimillonarios que se licitan en el mercado internacional. Por tal panorama, es de coincidir en la opinión de Balderas (2013), quién opina que la competencia entre empresas y sus productos, tiene una dimensión global de economías que son interdependientes; inclusive, Balderas (2013) relaciona sus comentarios del punto anterior con el concepto de la aldea global de McLuhan \& Powers (1992).

Uno de los principales factores que determinan quién se llevará los fondos que representan los contratos públicos y privados, es el precio de venta. Un bajo-costo es un diferenciador para que pequeñas compañías locales puedan incursionar rápidamente al ámbito de los competidores 
globales (Sirkin et al., 2008). Así, el que una empresa sea eficiente en costos, puede hacer la gran diferencia, al menos en un inicio, entre tomar ventaja de la globalización o bien, ser desplazada por los competidores provenientes de todos lados del mundo. Un ejemplo de esto se puede observar con el caso de la India en la industria del software, en donde el gobierno promueve ventajas fiscales y políticas tributarias que permitan a su país seguir compitiendo en el entorno macroeconómico de los negocios (Soto, Jiménez, Galindo, \& Osorio, 2013).

En el caso de las empresas mexicanas -especialmente las prestadoras de servicios-, una de las formas para reducir los costos operativos es el diseño de estrategias de compensación laboral orientadas en reducir la carga fiscal de las personas morales, logrando así abaratar sus costos de capital de trabajo y, por ende, ofrecer precios competitivos en el mercado internacional. Si bien, estas estrategias logran el objetivo de reducir los costos de las empresas, cabe la siguiente interrogante, ¿existe un impacto adverso a largo plazo que actúa en detrimento del futuro financiero de los empleados, derivado de dichas estrategias? Y si es así, ¿están conscientes los empleados de cómo su actual esquema de compensación puede estar dejando un hueco importante en sus finanzas futuras?

Considerando que el objetivo de la empresa transnacional es la maximización de los ingresos a nivel del grupo (Trajtenberg, 1999), se puede entender la importancia para éstas de competir en la búsqueda de fondos y contratos, y es precisamente en la búsqueda de ventajas sobre los competidores, en donde las empresas transnacionales fijan como meta el ofrecer precios de venta atractivos, para lo cual requieren llevar a cabo estrategias de reducción y optimización de costos.

Cuando se trata de empresas cuya oferta al mercado se basa en el aprovisionamiento de servicios, algunas de las vías para la búsqueda de la eficiencia, generalmente van orientadas en los siguientes dos rubros:

1) Incrementar la producción o generación del bien o valor comercializable, a través de procesos más rápidos y que requieren menos esfuerzo. 
2) Reducción de los costos fijos y variables para generar el bien o valor comercializable.

De acuerdo con Angulo (2011), para alcanzar el objetivo de reducir costos, se puede optar por la implementación de una estrategia fiscal, la cual —idóneamente - tendría por objetivo al menos alguno de los siguientes:

- Diferimiento de los impuestos por pagar.

- Recuperación de los saldos a favor.

- Cumplimiento correcto de las obligaciones fiscales.

- Certeza para planear en términos financieros.

El objetivo de este estudio es conocer si las personas morales mexicanas con operaciones en el extranjero implementan acciones fiscales, que deriven en detrimento del futuro financiero de los empleados de estas empresas. La diversificación de conceptos que se consideren para retribuir a un trabajador por su trabajo puede tener consecuencias para la empresa como para sus trabajadores.

A partir de la información recabada, se busca plasmar un panorama de la relación entre actores económicos que se desencadena por la búsqueda de la competitividad empresarial y sus medidas adoptadas. El entender como algunas medidas de estrategia están ligadas al futuro patrimonial de un empleado, puede ayudar a que se formulen mejores estrategias, que no tengan un efecto adverso para el trabajador al largo plazo.

Este trabajo comienza con una revisión de la literatura para aclarar los términos más importantes que brindan el contexto sobre el que se posiciona el propósito a estudiar. Después, para ilustrar, se darán algunos ejemplos de medidas derivadas de estrategias fiscales, que impactan directamente en la forma de retribuir al trabajador.

Para poder contar también con una visión más nítida de lo que las empresas de hoy en día están haciendo para reducir sus costos, se diseñó una encuesta como instrumento para obtener información sobre el modelo de compensación de una muestra de trabajadores del sector empresarial en México. 


\section{Marco conceptual}

Para entender mejor a las empresas que enmarcan la competencia en los mercados internacionales, podemos hacer referencia al trabajo de Raúl Trajtenberg (1999), en el cual aborda a profundidad el concepto y las características de las empresas transnacionales, que son aquellas que cuentan con actividades productivas en más de un país. Entre las principales características que Trajtenberg identifica para estas empresas están:

- Gran volumen de operaciones

- Separación de la propiedad y el control

- Comportamiento oligopólico

- Constitución de grupos económicos

Ahora bien, para llevar a cabo sus operaciones, toda empresa requiere de personas que ejecuten ciertas tareas o actividades, y es aquí cuando se origina la necesidad de emplear personas que realicen el trabajo propio de la actividad empresarial. El artículo $8^{\circ}$ de laLey Federal del Trabajo de México (publicada en 1970 y reformada en 2015, p. 3), estipula:

Trabajador es la persona física que presta a otra, física o moral, un trabajo personal subordinado. Para los efectos de esta disposición, se entiende por trabajo toda actividad humana, intelectual 0 material, independientemente del grado de preparación técnica requerido por cada profesión u oficio. (Cámara de Diputados,1970)

Para el trabajador de una empresa, la compensación se puede percibir como un intercambio con el empleador por el trabajo realizado, como un derecho por ser un empleado de la compañía o bien, como un incentivo por el trabajo bien realizado (Milkovich, Newman, \& Gerhart, 2014). También, es importante tomar en cuenta lo que Varela (2006) refiere sobre el impacto directo en el nivel de vida que tiene el pago de una compensación para la mayoría de las personas, ya que esto da muestra de lo relevante que es el tener un modelo de compensación que satisfaga las necesidades y las aspiraciones de los empleados. 
En México, el Instituto Mexicano del Seguro Social (IMSS) es la institución que tiene a su cargo el diseño del esquema de compensación que sirve de base para determinar el pago a los trabajadores, y esto queda detallado en el artículo 286 I. de la Ley del Seguro Social (publicada en 1995 $y$ reformada en 2015, p.8) que establece que:

El Instituto conformará su estructura orgánica y ocupacional de acuerdo con las necesidades del servicio. Asimismo, diseñará y establecerá el sistema de compensación que servirá de base para determinar el pago de remuneraciones, prestaciones y estímulos en favor de los trabajadores de confianza a que se refiere el artículo 256 de esta Ley, con el fin de mantener su competitividad en el mercado laboral. (Cámara de Diputados, 1995).

La compensación a los trabajadores debe vislumbrarse con tres componentes: el primero es la forma en que se administran los sueldos y salarios; el segundo, las prestaciones, incentivos y pagos variables a los que un empleado es acreedor; $y$ el tercero, la forma en que se paga al personal, esto en términos contables y fiscales (Varela, 2006).

Dentro del ámbito de Recursos Humanos, Milkovich et al., (2014) refieren la existencia de lo que llaman mejores prácticas en compensación, que son aquellas que están alineadas a recompensar a los empleados identificados como indispensables para cumplir con éxito el plan estratégico de la compañía. Estas mejores prácticas pueden variar en función de la empresa, el tipo de industria, el tamaño de la misma e inclusive la cultura que predomina en la región. Lo que es importante, de acuerdo con la opinión de varios autores, es contar con un modelo que permita incentivar al empleado no sólo por su trabajo, sino también por su lealtad, generación de valor, innovación, entre otros aspectos.

Ahora bien, desde la perspectiva contable, la compensación tiene implicaciones para cualquier organización, ya que con base en ella se definirá una importante parte de la carga fiscal. Sánchez Vega (2009, citado en Angulo, 2011) señala que una estrategia fiscal tendrá por objeto la

\section{Tolentino}


optimización de la carga tributaria, siempre dentro del más absoluto respeto a los preceptos legales relativos, a efecto de prever los resultados financieros y fiscales, sin ubicarse en los supuestos delictivos.

También es pertinente hacer referencia al antecedente de nuestro país en materia tributaria, ya que históricamente México ha presentado niveles bajos de recaudación por diversas causas; desde la elusión, que consta de aprovechar los huecos legales para evitar la generación de obligaciones fiscales, hasta la evasión que conlleva a una violación directa a la legislación fiscal (Hinojosa-Cruz, 2012). Precisamente como parte de la cultura de los contribuyentes de México (y América Latina casi en su mayoría), es el justificar la elusión fiscal como respuesta a la evidente corrupción en las instituciones del estado (Daude, Gutiérrez, \& Melguizo, 2012).

Los modelos de compensación están orientados en proveer a los trabajadores de una empresa con la retribución e incentivos por su trabajo. La empresa debiera definir estos modelos con base en estrategias que: cubran las necesidades de los empleados, sean costeables, permitan la generación de utilidades y no generen un alto pasivo laboral (Milkovich et al., 2014; Varela, 2006).

\section{Métodos utilizados}

Este estudio inicial se enmarca en una investigación más amplia que utilizará un diseño de métodos mixtos, siguiendo lo que proponen autores como Creswell y Plano Clark (2011) para proyectos que involucran la recolección y tratamiento de datos tanto cuantitativos, como cualitativos.

En la primera etapa que aquí se presenta, se llevó a cabo bajo un enfoque cuantitativo para obtener información inicial que permitiera esbozar un panorama sobre los modelos de compensación utilizados por las empresas en el país. Por ello, en principio se llevó a cabo una revisión de literatura en temas de compensación laboral para identificar los principales conceptos teóricos, regulaciones fiscales y leyes aplicables al trabajo remunerado; esto, con la finalidad de enmarcar la situación a estudiar. 
Después, haciendo uso de la información recabada y la experiencia dentro del ámbito laboral empresarial, se diseñó una encuesta como instrumento de medición para abordar la situación actual en términos de compensación y beneficios de los empleados de empresas en México. Esta encuesta estaba dividida en las siguientes secciones: Política de privacidad, Datos generales, Información del empleador actual, Condiciones laborales, Prestaciones sociales, compensación, prestaciones y beneficios, Ahorro para el retiro y Ánimo laboral en función de la compensación.

Debido al tamaño de la población de trabajadores en empresa de los sectores secundarios y terciarios en el país, así como del objetivo de este estudio, se optó por realizar un muestreo de bola de nieve a través de 5 informantes iniciales inmersos en el trabajo empresarial, cuyas variables de interés debían cumplir con las siguientes características:

- Nacionalidad mexicana

- Laborando actualmente

- Contratación por una empresa con operaciones transnacionales

A estos primeros informantes se les aplicó la encuesta diseñada, misma que compartieron - con sus contactos de características similares- a través de redes y medios sociales tales como Facebook, Linkedln y WhatsApp. El muestreo se realizó del $1^{\circ}$ de octubre al 15 de noviembre del 2016; al cierre del mismo, se recibieron un total de 45 encuestas electrónicas respondidas.

De la muestra que participó respondiendo la encuesta, el $77.78 \%$ expresó estar laborando actualmente, con una distribución que se muestra en la Tabla 1.

Tabla 1. Situación laboral de los encuestados

\begin{tabular}{lccc}
\hline Situación Laboral & Hombres & Mujeres & Prefiere No Decir \\
\hline Actualmente laborando & $44.44 \%$ & $31.11 \%$ & $2.22 \%$ \\
Actualmente no está laborando & $8.89 \%$ & $13.33 \%$ & - \\
Total & $53.33 \%$ & $44.44 \%$ & $2.22 \%$ \\
\hline
\end{tabular}

Fuente: Elaboración del autor. 
La Tabla 2 muestra la distribución de los encuestados que se encuentran laborando actualmente, en función del mercado en donde opera la empresa, así como el tipo de actividad económica de la misma. La mayor parte de los encuestados que actualmente trabajan, lo hacen para una empresa que presta servicios, ya sea de forma nacional o también con un enfoque internacional.

Tabla 2. Encuestados por tipo de empresa para la que laboran.

\begin{tabular}{lcc}
\hline \multicolumn{1}{c}{ Empresas } & $\begin{array}{c}\text { Prestadora de } \\
\text { Servicios }\end{array}$ & $\begin{array}{c}\text { Productora y/o } \\
\text { Comercializadora }\end{array}$ \\
\hline Con operaciones en México & $31.43 \%$ & $25.71 \%$ \\
Con operaciones en México y el Extranjero & $37.14 \%$ & $5.71 \%$ \\
Total & $68.57 \%$ & $31.43 \%$ \\
\hline
\end{tabular}

Fuente: Elaboración del autor.

En el caso de las personas que laboran para empresas transnacionales, sólo un $75 \%$ respondió que el salario percibido se encuentra íntegramente reportado ante el IMSS.

Figura 1. Resultado de la pregunta sobre el salario registrado ante el IMSS

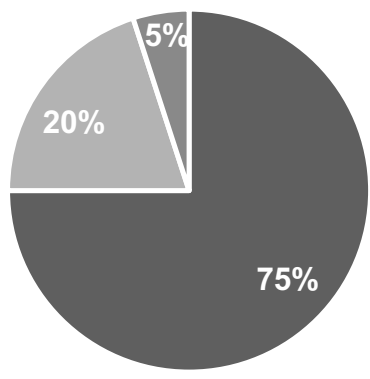

- Corresponde al salario que recibo por parte de mi empleador

- Es menor al salario que recibo por parte de mi empleador

- No estoy seguro

Fuente: Elaboración del autor.

También, es importante mencionar que, de estos trabajadores encuestados, solo un 45\% expresó conocer la ley bajo la cual se realizará su proceso de jubilación. Un dato aún más relevante es que, de la muestra de 
encuestados, únicamente 2 personas expresaron que sí realizan aportaciones voluntarias en la cuenta de su Administradora de Fondos para el Retiro (AFORE).

Figura 2. Conocimiento de la ley bajo la cual se realizará su jubilación

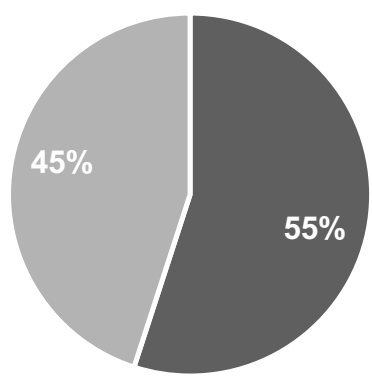

- Conoce la ley aplicable para su jubilación

- No conoce la ley aplicable para su jubilación

Fuente: Elaboración del autor.

La información recabada permite observar un panorama general sobre los modelos de compensación que están siendo diseñados y aplicados por las empresas, para luego hacer un análisis con respecto al contexto del ahorro para el retiro de los trabajadores. Un ejemplo es el registro del salario correspondiente del trabajador ante el Seguro Social por parte de los empleadores. Del grupo de encuestados que laboran en empresas transnacionales, hay un $20 \%$ de trabajadores que tienen registrado ante el Instituto Mexicano del Seguro Social un salario menor al que realmente perciben. Además, un $5 \%$ de los encuestados ni siquiera sabe qué salario tiene registrado ante dicha institución.

\section{Análisis de la situación actual en México}

En nuestro país, la más reciente Ley del Seguro Social (1995) es la que establece los criterios para el retiro por cesantía y vejez de los trabajadores. También, en sus estatutos, se asigna a la Comisión Nacional del Sistema de 
Ahorro para el Retiro (CONSAR), la atribución de dictar los requisitos con los que deberán contar los planes de pensiones en México.

Las aportaciones que se realizan de manera periódica al Fondo del Retiro de un trabajador, están determinadas por la Ley en función del salario integrado ante el Seguro Social y se componen de la siguiente manera, según lo muestra el portal de la Comisión Nacional del Sistema de Ahorro para el Retiro - (CONSAR, 2016):

- $\quad 1.125 \%$ lo aporta el trabajador (se descuenta de forma obligatoria).

- $\quad 5.150 \%$ lo aporta el empleador.

- $\quad 0.225 \%$ lo aporta el gobierno.

De acuerdo con lo anterior, el ahorro obligatorio que se reserva para el retiro de los trabajadores suma apenas un $6.5 \%$ del salario mensual con el que se cuenta. Además, vale la pena explicar que, si bien un trabajador puede recibir una compensación de cierto monto por parte de su empleador, dicha compensación está compuesta por diversos conceptos, y no todos forman parte del salario integrado que se utiliza en el cálculo del Ahorro para el Retiro. Para lo anterior, nos podemos remitir al artículo 27 de la Ley del Seguro Social (1995, pp. 10-11), que enuncia lo siguiente:

Artículo 27. El salario base de cotización se integra con los pagos hechos en efectivo por cuota diaria, gratificaciones, percepciones, alimentación, habitación, primas, comisiones, prestaciones en especie y cualquiera otra cantidad o prestación que se entregue al trabajador por su trabajo. Se excluyen como integrantes del salario base de cotización, dada su naturaleza, los siguientes conceptos:

I. Los instrumentos de trabajo tales como herramientas, ropa y otros similares;

II. El ahorro, cuando se integre por un depósito de cantidad semanaria, quincenal o mensual igual del trabajador y de la empresa; si se constituye en forma diversa o puede el trabajador retirarlo más de dos veces al año, integrará salario; tampoco se 
tomarán en cuenta las cantidades otorgadas por el patrón para fines sociales de carácter sindical;

III. Las aportaciones adicionales que el patrón convenga otorgar a favor de sus trabajadores por concepto de cuotas del seguro de retiro, cesantía en edad avanzada y vejez;

IV. Las cuotas que en términos de esta Ley le corresponde cubrir al patrón, las aportaciones al Instituto del Fondo Nacional de la Vivienda para los Trabajadores, y las participaciones en las utilidades de la empresa;

V. La alimentación y la habitación cuando se entreguen en forma onerosa a los trabajadores; se entiende que son onerosas estas prestaciones cuando el trabajador pague por cada una de ellas, como mínimo, el veinte por ciento del salario mínimo general diario que rija en el Distrito Federal;

VI. Las despensas en especie o en dinero, siempre y cuando su importe no rebase el cuarenta por ciento del salario mínimo general diario vigente en el Distrito Federal;

VII. Los premios por asistencia y puntualidad, siempre que el importe de cada uno de estos conceptos no rebase el diez por ciento del salario base de cotización;

VIII. Las cantidades aportadas para fines sociales, considerándose como tales las entregadas para constituir fondos de algún plan de pensiones establecido por el patrón o derivado de contratación colectiva. Los planes de pensiones serán sólo los que reúnan los requisitos que establezca la Comisión Nacional del Sistema de Ahorro para el Retiro, $y$

IX. El tiempo extraordinario dentro de los márgenes señalados en la Ley Federal del Trabajo.

Para que los conceptos mencionados en este precepto se excluyan como integrantes del salario base de cotización, deberán estar debidamente registrados en la contabilidad del patrón. En los conceptos previstos en 
las fracciones VI, VII y IX cuando el importe de estas prestaciones rebase el porcentaje establecido, solamente se integrarán los excedentes al salario base de cotización. (Cámara de Diputados ,1995)

Un ejemplo de lo anterior es el caso de un trabajador de una empresa transnacional de la industria del software que recibe una compensación que se detalla en la Tabla 3.

Tabla 3. Desglose de compensación de un empleado en una empresa de software con operaciones en México y el extranjero

\begin{tabular}{lcr}
\hline Percepciones & Compensación en pesos mexicanos \\
\hline Compensación Fija Mensual & $\$ 45,500.00$ \\
Salario & $\$$ & $18,200.00$ \\
Premio de Asistencia & $\$$ & $1,820.00$ \\
Premio de Puntualidad & $\$$ & $1,820.00$ \\
Vales de Despensa & $\$$ & 807.48 \\
Actividades Culturales & $\$$ & $2,018.70$ \\
Fomento Ahorro & $\$$ & $7,522.00$ \\
Derechos de Autor & $\$$ & $9,733.03$ \\
Total de Percepciones & $\$$ & $41,921.21$ \\
Retenciones & & \\
ISR & & \\
IMSS & $\$$ & $3,768.93$ \\
Bono de Diciembre & $\$$ & 503.66 \\
Apoyo para el Retiro & $\$$ & $1,527.05$ \\
Total de Retenciones & $\$$ & 529.55 \\
& & $\$$ \\
\hline
\end{tabular}

Fuente: Elaboración del autor con información compartida por uno de los informantes encuestados.

Se pueden observar, en el primer apartado, varios conceptos que suman un total de percepciones mensuales por $\$ 41,921.21$ pesos para el trabajador. Pero considerando las restricciones que establece el artículo 27 de la Ley del Seguro Social, los conceptos por Premio de Asistencia, Premio de Puntualidad, Vales de Despensa, Actividades Culturales, Fomento Ahorro y Derechos de Autor, no forman parte del Salario Diario Integrado de un trabajador, por lo que la aportación al Fondo para el Retiro de dicho 
trabajador es calculada sobre el Salario de $\$ 18,200.00$ pesos. Esto en los números que brinda la Ley, se traduce en un monto mensual de Ahorro para el Retiro de este empleado, por la cantidad de $\$ 1,183.00$ pesos.

\section{Resultados del estudio}

Del grupo de encuestados que participaron en este estudio y que mencionaron estar laborando para una empresa transnacional, el 90\% aceptó que no realiza aportaciones voluntarias para su retiro. Cabe mencionar que la edad promedio de los encuestados es de 31 años (el rango de edad de los encuestados está entre los 22 y los 56 años) lo que no justifica que estemos tratando con personas de reciente inmersión en el mundo laboral. Incluso, el promedio de tiempo laborando con el actual patrón entre los encuestados, es de casi 4 años.

También, vale la pena resaltar que, en lo referente a la escala de satisfacción con el actual modelo de compensación laboral, el $45 \%$ de los encuestados expresó estar en los niveles más altos de satisfacción. Y esto pareciera no importar al $20 \%$ de los encuestados cuyo salario registrado ante el IMSS es menor al que reciben por parte de su empleador.

De acuerdo con los datos obtenidos de este estudio, las empresas que están participando del escenario internacional (con operaciones también en el extranjero), están brindando beneficios adicionales dentro de sus paquetes de compensaciones, como por ejemplo acceso a fondo de ahorro, vales de despensa y vales de gasolina, entre otros, tal como se puede observar en la Figura 3. Adicionalmente, el $43 \%$ de encuestados que trabajan y tienen registrado su sueldo íntegro ante el Seguro Social, expresó además que cuenta con un contrato de trabajo por tiempo indefinido. 
Figura 3. Conceptos que conforman la compensación laboral en los encuestados de empresas transnacionales

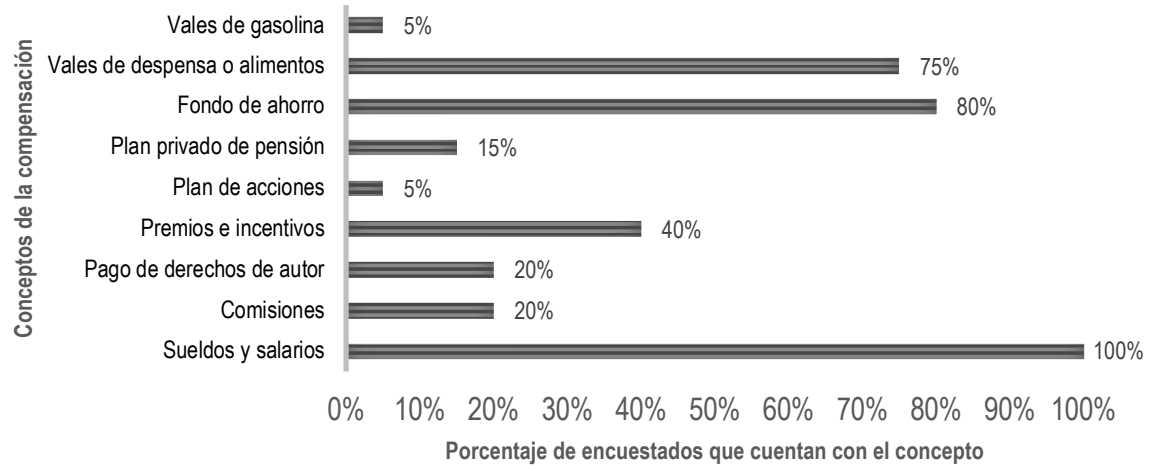

Fuente: Elaboración del autor.

En la muestra que respondió, se observa que hay poco o nulo conocimiento sobre el Seguro Social, las leyes que derivan de esta institución y lo referente al Sistema de Ahorro para el Retiro que existe en México.

También se puede dejar en evidencia como algunos modelos de compensación laboral que son atractivos por el monto que se paga al empleado, pueden estar conformados por diversos conceptos que a la larga dejarán un "vacío" en la previsión económica futura del trabajador.

\section{Conclusiones}

De acuerdo con los resultados de este estudio, efectivamente se están aplicando estrategias fiscales en la definición de los modelos de compensación de los empleados, por parte de las empresas transnacionales. Estas estrategias funcionan perfectamente para incentivar a los empleados, a su vez que la empresa reduce el monto de sus obligaciones fiscales. Como lo ha expuesto Toledo-Espinosa (2012), en México hay toda una gama de opciones de compensación en especie. Algunas de las principales que 
atribuyen el beneficio fiscal de reducción de la base gravable para el pago de Impuesto Sobre la Renta (ISR) incluyen:

- Prestaciones en acciones de la compañía

- Adquisición o arrendamiento de automóvil

- Apoyo a la salud del empleado (Seguro de Gastos Médicos, Seguro de Vida, acceso a clubes deportivos)

En el caso de que ciertos beneficios de la compensación, tales como los seguros médicos 0 de vida, se brinden a la totalidad de empleados 0 colectividad específica (por ejemplo un sindicato), estos pueden caer bajo el rubro de previsión social para efectos del cálculo del pago de Impuesto Sobre la Renta de acuerdo con el artículo $7^{\circ}$, y por ende son deducibles para las personas morales de acuerdo a lo establecido en el artículo 27 de la misma ley (Cámara de Diputados, 2013).

Una empresa que tenga una verdadera ética salarial para con sus empleados, puede encontrar opciones como las arriba mencionadas, antes de tener que armar un modelo de compensación que esquive la Ley Federal del Trabajo, limitando derechos de prestaciones sociales básicas. Un claro ejemplo de falta de ética salarial se pone de manifiesto cuando el empleado es registrado ante el IMSS con un salario menor al pagado por la empresa.

Los trabajadores de hoy en día no están al tanto de las leyes aplicables en materia laboral, de previsión social ni de retiro. Cobijarse con lo que establecen las distintas leyes y, sobre todo, los derechos como trabajadores, pueden ser un diferenciador entre estar preparado para el futuro o bien, llevarse una sorpresa financiera muy desagradable.

\section{Referencias}

Angulo, E. (2011). Politica fiscal y estrategia como factor de desarrollo de la mediana empresa comercial Sinaloense. Un estudio de caso, Tesis Doctoral, Culiacán: Universidad Autónoma de Sinaloa.

Balderas, F. de J. (2013). La multidimensionalidad del salario aproximación de un modelo de salarios equitativos para México (1a ed.). Monterrey: Editorial Liblio.

Cámara de Diputados. (1970). Ley federal del trabajo. Diario Oficial de la Federación, 1 de abril de 1970. Estados Unidos Mexicanos: Diario Oficial de la Federación. 
Cámara de Diputados. (1995). Ley del seguro social. Diario Oficial de la Federación, 21 de diciembre de 1995. Ciudad de México: Diario Oficial de la Federación.

Cámara de Diputados. (2013). Ley del impuesto sobre la renta. Diario Oficial de La Federación. Ciudad de México: Diario Oficial de la Federación.

CONSAR. (2016). Comisión Nacional del Sistema de Ahorro para el Retiro.

Creswell, J. W., \& Plano Clark, V. L. (2011). Designing and conducting mixed methods research (2nd ed.). Thousand Oaks, California: Sage.

Daude, C., Gutiérrez, H., \& Melguizo, Á. (2012). What drives Tax Morale? OECD Development Centre Working Papers, (315), 49.

Hinojosa-Cruz, A. V. (2012). La elusión fiscal y la responsabilidad social empresarial en México : El IETU como impuesto de control, Universidad Autónoma de Nuevo León Resumen. Monterrey.

McLuhan, M., \& Powers, B. R. (1992). The global village. Reino Unido: Oxford University.

Mendieta, G. (2015). Informantes y muestreo en investigación cualitativa. Investigaciones Andina, 17(30), 1148-1150.

Milkovich, G., Newman, J., \& Gerhart, B. (2014). Compensation (11th ed.). McGraw-Hill.

Sirkin, H. L., Hemerling, J. W., Bhattacharya, A. K., \& Butman, J. (2008). Globality competing with everyone from everywhere for everything. New York.

Soto, I., Jiménez, M., Galindo, J. \& Osorio, J. (2013). Key factors analyses that take software industry of India to succeed, Innovaciones de Negocios, 10(19), 37-64.

Toledo-Espinosa, M. (2012). Plan de prestaciones en especie para ejecutivos. Veritas.

Trajtenberg, R. (1999). El concepto de empresa transnacional.

Varela, R. A. (2006). Administración de la compensación. Sueldos, salarios y prestaciones. (J. Reyes-Martinez, Ed.). Estado de México: Pearson Educación. 